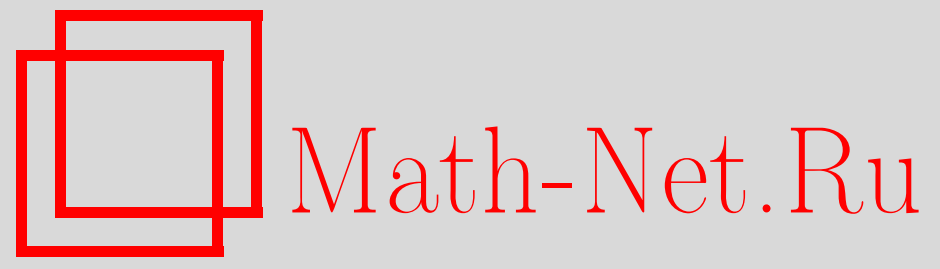

К. К. Шукин, Об изоморфизме квазигрупп, Дискрет. матем., 2005, том 17, выпуск 3, 109-111

DOI: https://doi.org/10.4213/dm120

Использование Общероссийского математического портала Math-Net.Ru подразумевает, что вы прочитали и согласны с пользовательским соглашением http: //www.mathnet.ru/rus/agreement

Параметры загрузки:

IP : 54.237 .206 .68

26 апреля 2023 г., 16:00:26

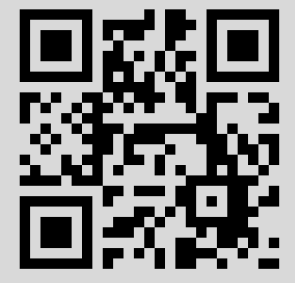


Удк 512.6

\title{
Об изоморфизме квазигрупп
}

\author{
() 2005 г. К. К. Щукин
}

В этой заметке представлено решение известной задачи об изоморфизме квазигрупп, каждая из которых главно изотопна одной и той же квазигруппе.

Справедливо следующее утверждение.

Предложение 1. Пусть $(\lambda, \mu, \varepsilon) u(\varphi, \varphi, \varphi)$ - произвольные главная изотопия и изоморфизм квазигруппь $(Q, \cdot)$.

(1) Условия $\varphi=\psi, \alpha=\varphi \lambda \varphi^{-1}, \beta=\varphi \mu \varphi^{-1}$ являются достаточными для коммутативности диаграммы

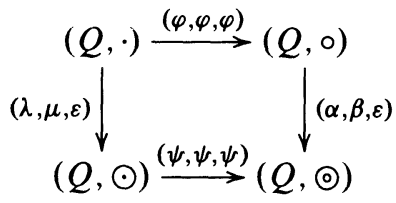

Эти условия остаются и необходимыми, если только

$$
\left(\varphi^{-1} \alpha^{-1} \psi \lambda, \varphi^{-1} \beta^{-1} \psi \mu, \varphi^{-1} \psi\right)
$$

является единичной автотопией квазигруппы $(Q, \cdot)$.

(2) Если $(\alpha \varphi, \beta \varphi, \varphi)$ является единичной автотопией квазигруппы $(Q, \cdot)$, то коммутативность диаграммы

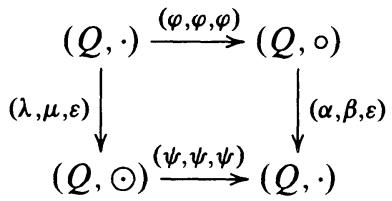

порождает автотопию $(\psi \lambda, \psi \mu, \psi)$ квазигруппы $(Q, \cdot)$. Обратно, каждая пара автотопий $(\alpha \varphi, \beta \varphi, \varphi)$ и $(\psi \lambda, \psi \mu, \psi)$ квазигруппы $(Q, \cdot)$ порождает ее коммутативную диаграмму указанного выше типа. При условиях $\varphi=\psi, \alpha=\varphi \lambda \varphi^{-1}$, $\beta=\varphi \mu \varphi^{-1}$ и дополнительном требовании $\varphi^{2}=\varepsilon$ появляется также автотопия

$$
\left(\lambda \varphi^{-1}, \mu \varphi^{-1}, \varphi^{-1}\right)
$$

квазигрупn $(Q, \circ)=(Q, \odot)$. 
Доказательство. Докажем (1). Диаграмма предполагает, что

$$
\begin{aligned}
\varphi(x y) \varphi(x) \circ \varphi(y) & =\alpha \varphi(x) \odot \beta \varphi(y), \\
\psi(x y)=\psi(\lambda(x) \odot \mu(y)) & =\psi \lambda(x) \odot \psi \mu) y)
\end{aligned}
$$

для любых $x, y \in Q$. Предполагая, что $\varphi=\psi, \alpha=\varphi \lambda \varphi^{-1}, \beta=\varphi \mu \varphi^{-1}$, получаем равенство

$$
(\alpha, \beta, \varepsilon)(\varphi, \varphi, \varphi)=(\varphi \lambda, \varphi \mu, \varphi)=(\psi, \psi, \psi)(\lambda, \mu, \varepsilon)
$$

которое определяет коммутативность диаграммы. Наоборот, исходя из коммутативности диаграммы $(\alpha \varphi, \beta \varphi, \varphi)=(\psi \lambda, \psi \mu, \psi)$, запишем равносильное равенство

$$
(\varepsilon, \varepsilon, \varepsilon)=\left(\varphi^{-1} \alpha^{-1} \psi \lambda, \varphi^{-1} \beta^{-1} \psi \mu, \varphi^{-1} \psi\right)
$$

Отсюда, равенства $\varphi=\psi, \alpha=\varphi \lambda \varphi^{-1}, \beta=\varphi \mu \varphi^{-1}$ выполняются только в том случае, когда $x \cdot y=\psi^{-1} \varphi\left(\varphi^{-1} \alpha^{-1} \psi \lambda x \cdot \varphi^{-1} \beta^{-1} \psi \mu y\right)$ является единичной автотопией квазигруппы $(Q, \cdot)$.

Докажем (2). Первая часть утверждения следует из строения диаграммы в (2), а также очевидного представления изотопии в виде произведения изоморфизма и главной изотопии. Далее, полагая $\varphi=\psi, \alpha=\varphi \lambda \varphi^{-1}, \beta=\varphi \mu \varphi^{-1}$, получаем автотопию $(\varphi \lambda, \varphi \mu, \varphi)=(\alpha \varphi, \beta \varphi, \varphi)$ квазигруппы $(Q, \cdot)$, изоморфной $(Q, \circ)$.

Отсюда трансформированием получаем автотопию квазигруппы $(Q, \circ)$

$$
(\varphi, \varphi, \varphi)(\varphi \lambda, \varphi \mu, \varphi)(\varphi, \varphi, \varphi)^{-1}=\left(\varphi^{2} \lambda \varphi^{-1}, \varphi^{2} \mu \varphi^{-1}, \varphi\right) .
$$

Если $\varphi^{2}=\varepsilon$, то получаем объявленную в утверждении (2) автотопию

$$
\left(\lambda \varphi^{-1}, \mu \varphi^{-1}, \varphi^{-1}\right)=(\lambda, \mu, \varepsilon)\left(\varphi^{-1}, \varphi^{-1}, \varphi^{-1}\right),
$$

в частности, $(Q$, о $)=(Q, \odot)$.

Следствие 1. Если изотопия $(\alpha \varphi, \beta \varphi, \varphi)$ не является автотопией квазигруппы $(Q, \cdot)$, то диаграмма

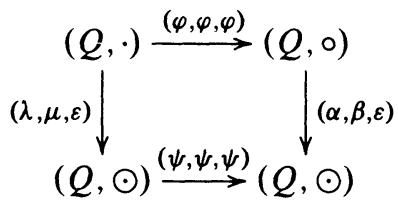

является коммутативной при условии, что $\varphi=\psi \in \operatorname{Aut}(Q, \odot), \alpha=\varphi \lambda \varphi^{-1}$, $\beta=\varphi \mu \varphi^{-1}$.

Доказательство следует из утверждений (1) и (2) с учетом специфики строения диаграммы следствия.

Заметим, что, в частности, мы получаем решение задачи об изоморфизме квазигрупп $(Q, \cdot)$ и $(Q, \circ)$, главно изотопных одной и той же группе $(Q, \odot)$. Эта проблема рассматривалась в [2]. 


\section{Список литературы}

1. Shchukin K. K., On the isomorphisms of the quasigroups. In: Commun. 2nd Conf. Math. Soc. Moldova 2004, p. 292.

2. Izbash V. I., Isomorphisms of quasigroups isotopic to groups. Quasigroups and Related Systems (1995) 2, №1, 34-41.

Статья поступила 18.10.2004. 